\title{
A Generalized Soft-Sphere Model for Monte Carlo Simulation
}

\author{
Jing Fan \\ Institute of Mechanics, Chinese Academy of Sciences, Beijing 100080, China
}

\begin{abstract}
A new collision model, called the generalized soft-sphere (GSS) model, is introduced. It employs the same total cross section as the generalized hard-sphere (GHS) model, whereas the deflection angle is calculated by the soft-sphere scattering model. In virtue of a two-term formula given to fit the numerical solutions of collision integrals for the Lennard-Jones (6-12) potential and for the Stockmayer potential, the parameters of the GSS model are determined explicitly. Coefficients of viscosity, self-diffusion and diffusion predicted by the GSS model compare quite well with measured data for both polar and non-polar molecules over a wide temperature range.
\end{abstract}

\section{INTRODUCTION}

The direct simulation Monte Carlo (DSMC) method is a general numerical technique to analyze rarefied gas flows [1]. The strategy of DSMC is to track the molecular trajectory and status based on the collision mechanics of model molecules, and then obtain physical quantities of interest through statistical average. A physically realistic collision model is therefore critical for DSMC to predict various gas flows reliably and accurately. In actual gases the force between two molecules is repulsive at small distances, and more weakly attractive at larger distances. This behavior is most simply described by the Lennard-Jones (6-12) potential $[2,3]$

$$
\varphi(r)=4 \varepsilon\left[\left(\frac{\sigma}{r}\right)^{12}-\left(\frac{\sigma}{r}\right)^{6}\right] .
$$

The parameters $\varepsilon$ and $\sigma$ (which have dimensions of energy and length, respectively) are constants characteristic of the chemical species of the colliding molecules, and $r$ is the inter-molecular separation. Systematic studies [2,3] showed that the potential (1) was adequate for many non-polar molecules such as air.

For polar molecules like ammonia and water vapor, however, strong electrostatic interaction leads to anomalous behavior with respect to the potential (1) [2,3]. This electrostatic contribution is proven proportional to the inverse third power of the intermolecular separation. Stockmayer combined such a term with the Lennard-Jones potentia [2,3]

$$
\varphi(r)=4 \varepsilon\left[\left(\frac{\sigma}{r}\right)^{12}-\left(\frac{\sigma}{r}\right)^{6}-\delta\left(\frac{\sigma}{r}\right)^{3}\right] .
$$

where $\delta$ is a dimensionless constant that measures the polarization of a substance. The potential (2), known as the Stockmayer potential, was successfully applied to many polar molecules [2,3].

An accurate procedure to calculate collision cross sections and scatter under the potentials (1) or (2) has been widely used in kinetic studies of transport properties [2,3]. Application of this procedure to DSMC, though straightforward in principle, is too computationally intensive in engineering context where millions and even 
billions of molecular collisions have to be considered. Many efforts [4-9] have been paid to establish a collision model appropriate to the DSMC method. The variable hard-sphere (VHS) model proposed by Bird $[4,5]$ assumes that the total cross section varies like

$$
\sigma_{T} \propto c_{r}^{-2 \omega}
$$

while the deflection angle in a collision follows the hard sphere scattering law, i.e. an isotropic scattering in the center of mass frame of reference, where $c_{r}$ is the relative speed of the colliding molecules.

The total cross section (3) bears the same relation of coefficient of viscosity to temperature as the inverse-power-law (IPL) potential $[1,4]$

$$
\mu \propto T^{\omega+1 / 2} .
$$

It is known that IPL considers the repulsive force between molecules only and becomes poor at low temperatures where the attractive force dominates [2,3]. Therefore, it is desirable to establish a model based on potentials with both attraction and repulsion like (1). Under the hard-sphere scattering, the potential (1) has the following relation of coefficient of viscosity to temperature [9]

$$
\mu \propto \frac{T^{1 / 6+1 / 2}}{1+c_{o} T^{-1 / 2}},
$$

where $c_{O}$ is a constant. Clearly, the relations (4) and (5) are inconsistent. This led to the introduction by Hassan and Hash [9] of the GHS model that still employs the hard-sphere scattering law, but the total collision cross section is assumed as

$$
\frac{\sigma_{T}}{\sigma^{2}}=\sum \beta_{j}\left(\frac{E_{t}}{\varepsilon}\right)^{-\ell},
$$

where $E_{t}=\frac{1}{2} m_{r} c_{r}^{2}, m_{r}$ is the reduced mass of two colliding molecules, and $\beta_{j}$ and $\ell_{j}$ are constants that depend on the interaction potential and the molecular species under consideration.

Koura and his co-workers [6,7] showed that the hard-sphere scattering led to an incorrect ratio of the momentum to viscosity cross-section. They suggested a soft-sphere scattering model to calculate the deflection angle [7]

$$
\chi=2 \arccos \left[(b / d)^{1 / \alpha}\right],
$$

where $b$ is the miss-distance impact parameter, $d$ is the collision diameter, and $\alpha$ is chosen to match the real ratio of the momentum to viscosity cross section.

Besides the computational efficiency, these models aimed at phenomenological reproduction of transport coefficients of real gases. They were to some extent successful, whereas certain important issues such as molecular polarization remain to be settled. Moreover, the parameters involved in the GHS model were not explicitly expressed, and had to be numerically solved through the least-square fitting of experimental data of viscosity coefficient for simple gases and of binary diffusion coefficient for gas mixtures [8,9]. This is somewhat inconvenient for practical use, and sometimes even infeasible because the measurement of the diffusion 
coefficients is difficult to observe and experimental data are often not available [3,11].

In this paper, firstly, a universal collision model, named as generalized soft-sphere (GSS) model, is suggested that applies to both polar and non-polar molecules over a wide temperature range. Then, a fitting formula of the numerical solutions of the collision integrals for the potentials (1) and (2) is given, which is appropriate to determine the parameters of the GSS model explicitly. Next, transport coefficients predicted by GSS previous models are compared with experimental data.

\section{GSS MODEL}

The GSS model combines the advantages of the GHS and VSS models, in which the total cross section and the deflection angle are described by Eqs. (6) and (7), respectively. The main difficulty associated with this model is how to determine the involved parameters explicitly, which is essentially important for practical convenience.

It is known that the coefficients of viscosity and self-diffusion of a simple gas, to the first approximation, may be written as $[2,3]$

$$
\begin{aligned}
& \mu=\frac{5}{16}\left(\frac{\sqrt{\pi m k T}}{\pi \sigma^{2} \Omega^{(2,2)^{*}}}\right), \\
& D=\frac{3 k T}{16 p}\left(\frac{\sqrt{2 \pi k T / m_{r}}}{\pi \sigma^{2} \Omega^{(1,1)^{*}}}\right),
\end{aligned}
$$

with

$$
\begin{aligned}
& \Omega^{(1,1)^{*}}=\frac{1}{\pi \sigma^{2}} \int_{0}^{\infty} \exp \left(-\zeta^{2}\right) \zeta^{5} \sigma_{D} d \zeta, \\
& \Omega^{(2,2)^{*}}=\frac{1}{2 \pi \sigma^{2}} \int_{0}^{\infty} \exp \left(-\zeta^{2}\right) \zeta^{7} \sigma_{\mu} d \zeta,
\end{aligned}
$$

where $\zeta^{2}=\frac{1}{2} m_{r} c_{r}^{2} / k T, \sigma_{\mu}$ and $\sigma_{D}$ are the viscosity and momentum cross sections, respectively, $p$ and $T$ are the gas pressure and temperature, $m$ is the molecular mass, and $k$ is the Boltzmann constant. For the soft-sphere scattering, we have [7]

$$
\sigma_{\mu}=\frac{2 S_{\mu}}{3} \sigma_{T}, \sigma_{D}=S_{D} \sigma_{T}
$$

with

$$
\begin{gathered}
S_{\mu}=6 \alpha /[(\alpha+1)(\alpha+2)], \\
S_{D}=2 /(\alpha+1) .
\end{gathered}
$$

Substituting of Eqs. (6) and (12-14) into (10) and (11) yields the self-diffusion and viscosity integrals for the GSS model

$$
\Omega_{G S S}^{(1,1)^{*}}=\frac{1}{\pi\left(\alpha^{*}+1\right)} \sum \beta_{j}^{*} \Gamma\left(3-\ell_{j}^{*}\right) T_{*}^{-\ell_{j}^{*}}
$$




$$
\Omega_{G S S}^{(2,2)^{*}}=\frac{\alpha^{*}}{\pi\left(\alpha^{*}+1\right)\left(\alpha^{*}+2\right)} \sum \beta_{j}^{*} \Gamma\left(4-\ell_{j}^{*}\right) T_{*}^{-\ell_{j}^{*}}
$$

where $T_{*}=k T / \varepsilon$, and $\Gamma(\ldots)$ denotes the gamma function.

A simple means to determine the parameters of the GSS model is to compare the right-hand sides of Eqs. (15) and (16) against those of (10) and (11). However, the latter may be solved analytically only at special conditions such as IPL or the hard-sphere scattering law. For the Lennard-Jones (6-12) potential, there was only the numerical solution available, that was obtained by Hirschfelder, Curtiss and Bird and tabulated as functions of $T_{*}=k T / \varepsilon$ from 0.3 to 400 in Tables I-M, Ref. 3 . The current paper suggests a two-term fitting formula

$$
\Omega_{\text {fit }}^{(2,2)^{*}}=a_{1} T_{*}^{-c_{1}}+a_{2} T_{*}^{-c_{2}},
$$

where $a_{1}=1.1, a_{2}=0.4, c_{1}=0.133$, and $c_{2}=1.25$. As shown in Fig.1a), there is a remarkable agreement between Eq. (17) and the data of Hirschfelder, et al.

The fitting formula (17) is also suitable to the Stockmayer potential. $c_{1}$ and $c_{2}$ do not change, whereas $a_{1}$ and $a_{2}$ need an additional term to include the polarization effect

$$
a_{1}=1.1-0.044 \delta, \quad a_{2}=0.4+0.76 \delta
$$

In Fig. 1b), the formula (17), with $a_{1}$ and $a_{2}$ calculated by Eq. (18), compares well with the accurate solution obtained by Krieger [10] (also see Table IX, Ref. 3).

Equating the right hand sides of Eqs. (16) and (17) yields

$$
\begin{aligned}
& \ell_{1}^{*} \equiv c_{1}=0.133, \\
& \ell_{2}^{*} \equiv c_{2}=1.25,
\end{aligned}
$$

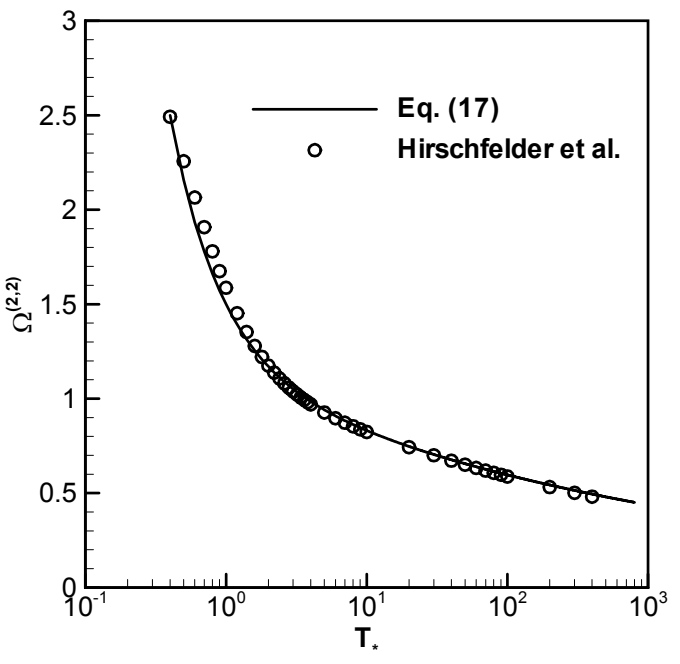

(a) Lennard-Jones (6-12) potential

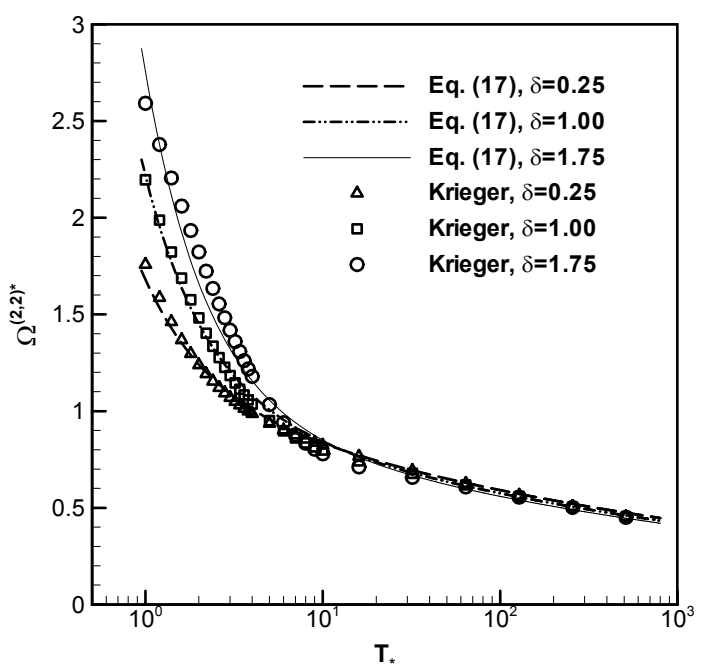

(b) Stockmayer potential

Figure 1 Comparison of the fitting formula (17) with the accurate solutions $[3,10]$. 


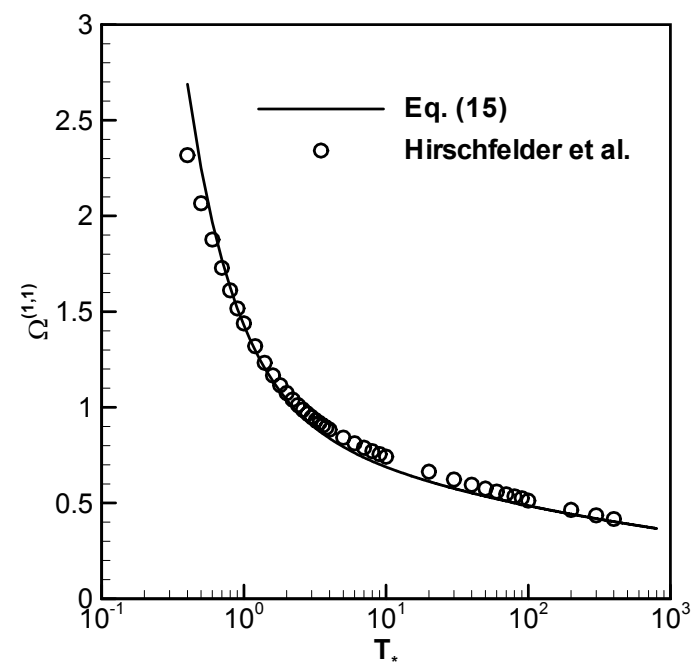

Figure 2 Comparison of the self-diffusion integral (15) for the GSS model with the accurate solution [3].

$$
\begin{aligned}
& \frac{\alpha^{*}}{\pi\left(\alpha^{*}+1\right)\left(\alpha^{*}+2\right)} \times \beta_{1}^{*} \Gamma\left(4-\ell_{1}^{*}\right) \equiv a_{1}, \\
& \frac{\alpha^{*}}{\pi\left(\alpha^{*}+1\right)\left(\alpha^{*}+2\right)} \times \beta_{2}^{*} \Gamma\left(4-\ell_{2}^{*}\right) \equiv a_{2},
\end{aligned}
$$

and $\beta_{j}^{*} \equiv 0$ for $j>2$. This indicates that the first two terms of the general form (6) are enough for either Lennard-Jones or Stockmayer potential.

Another relation to determine $\alpha^{*}, \beta_{1}^{*}$ and $\beta_{2}^{*}$ may be provided by $\Omega_{G S S}^{(1,1)^{*}}$ that is in satisfactory agreement with the numerical solution of $\Omega^{(1,1)^{*}}$ for the Lennard-Jones (6-12) potential given in Table I-M of Ref. 3, when (Fig. 3)

$$
\alpha^{*}=1.5 \text {. }
$$

This value is reasonable since $\alpha^{*}$ generally lies between 1 and 2 (cf. Ref. 1, pp.42).

From Eqs. (21-23), $\beta_{1}^{*}$ and $\beta_{2}^{*}$ may be easily solved

$$
\beta_{1}^{*}=3.962-0.158 \delta, \quad \beta_{2}^{*}=4.558+8.660 \delta .
$$

\section{$\varepsilon, \sigma$, and $\delta$}

The parameters $\varepsilon, \sigma$ and $\delta$ in the potentials (1) and (2) for like molecules may be determined from experimental data of coefficient of viscosity. A procedure was described in detail in Ref. 3 that also provided the values of $\varepsilon, \sigma$ and $\delta$ for many gases in Tables 8.4-1, 8.6-1 and I-A.

Force constants between unlike molecules, in principle, may be obtained from the binary diffusion coefficient at different temperatures. However, unlike the measurement of coefficients of viscosity that has been carried out accurately for a number of gases, few measurement of diffusion has been made [3,11]. It is customary 
to make use of the combining laws to relate the force constants between unlike molecules to those between like molecules [3]. If both unlike molecules are non-polar or polar, the combining laws are simply as

$$
\sigma_{i j}=\frac{\sigma_{i}+\sigma_{j}}{2}, \quad \varepsilon_{i j}=\sqrt{\varepsilon_{i} \varepsilon_{j}}, \quad \delta_{i j}=\sqrt{\delta_{i} \delta_{j}} .
$$

The interaction between a polar molecule and a non-polar molecule obeys the same potential as that between two non-polar molecules, i.e. the Lennard-Jone (6-12) potential, whereas the combining laws to determine the force constants are a little different [3]

$$
\sigma_{n p}=\frac{1}{2}\left(\sigma_{n}+\sigma_{p}\right) \xi^{-1 / 6}, \quad \varepsilon_{n p}=\sqrt{\varepsilon_{n} \varepsilon_{p}} \xi^{2}, \quad \xi=1+0.5 \alpha_{n} \sigma_{n}^{-3} \delta \sqrt{\varepsilon_{p} / \varepsilon_{n}},
$$

where $\sigma_{n}, \varepsilon_{n}$ are Lennard-Jones potential parameters for the non-polar molecule, $\sigma_{p}, \varepsilon_{p}$ are Stockmayer potential parameters for the polar molecule, and $\alpha_{n}$ is reduced polarizability of the non-polar molecule. The values of $\alpha_{n}$ for many gases were given in Table 13.2-3, Ref.3.

\section{COMPARISON OF MODELS WITH EXPERIMENT}

A number of experiments have been carried out to measure transport coefficients. The measured data obtained by different authors covered a wide temperature range, and were collected in Chapter 8, Ref. 3 and Table 6-177, Ref. 11. In the GSS model, the coefficients of viscosity, self-diffusion and diffusion are given by Eqs. (8), (9), (15) and (16), and the parameters involved are calculated by Eqs. (18-20), (23), and (24).

Figure 3a) compares the viscosity coefficient for argon given by the GSS model and experiment [3,11], which are in excellent agreement over the entire range of temperature. Also included is the profile given by the VSS model based on the IPL for which the VSS parameters are available (cf. Table I, [7], or Table A3, [1]). The VHS and GHS profiles, not shown in the figures, almost overlay with the VSS and GSS profiles, respectively. The differences between the VSS and experimental results at the low and high temperatures may be attributed to the attractive portion of molecular interaction neglected in IPL and a constant of $\omega$ used in VSS for convenience of engineering simulation. It is known that the attractive force becomes important at low temperature, and the real value of $\omega$ markedly varies with the range of temperatures (cf. [2], pp. 231).

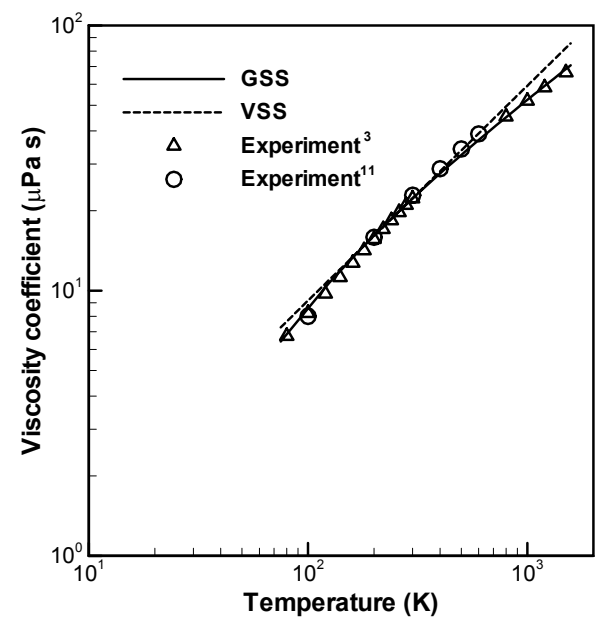

(a) $\mathrm{Ar}$

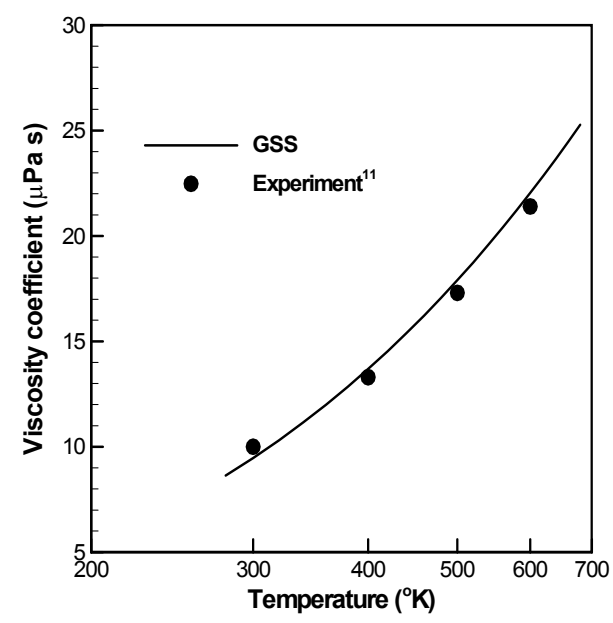

(b) $\mathrm{H}_{2} \mathrm{O}$

Figure 3 Comparison of coefficients of viscosity given by the GSS model with measured data [3,11]. 
The coefficients of viscosity of water vapor given by GSS and experiment [11] show a satisfactory agreement in Fig. 3b. Because of a strong polar property ( $\delta=2.333$ ), the IPL and Lennard-Jonese potentials are not suitable to this kind of molecules [2,3], so are the VHS, VSS and GHS models based on them.

Table 1 compares the self-diffusion coefficients for four common gases $\left(\mathrm{Ar}, \mathrm{H}_{2}, \mathrm{~N}_{2}\right.$, and $\left.\mathrm{CO}_{2}\right)$ given by the models and experiment [3]. The GSS results agree quite nicely with the measured data, with the difference generally within $5 \%$, except at an extremely low temperature of $20 \mathrm{~K}$ for hydrogen where it is nearly $10 \%$. The difference between the VSS model based on IPL and the experiment is about $10 \%$ at normal temperatures, but it becomes serious at the lower temperatures. Because of the hard-sphere scattering employed, the GHS and VHS results generally deviate more than $20-30 \%$ from the experimental data.

TABLE 1. Self-diffusion coefficients $\left(\mathrm{cm}^{2} \mathrm{sec}^{-1}\right)$ at $1 \mathrm{~atm}$ given by four models and experiment.

\begin{tabular}{ccccccc}
\hline Gas & $\mathrm{T}\left({ }^{\circ} \mathrm{K}\right)$ & GSS & VSS & GHS & VHS & Experiment [3] \\
\hline $\mathrm{Ar}$ & 77.7 & 0.0130 & 0.0178 & 0.0104 & 0.0134 & $0.0134 \pm 0.0002$ \\
& 273.2 & 0.161 & 0.173 & 0.129 & 0.131 & $0.156 \pm 0.002$ \\
& 353.2 & 0.258 & 0.276 & 0.206 & 0.207 & $0.249 \pm 0.003$ \\
$\mathrm{H}_{2}$ & 20.4 & 0.0100 & 0.0165 & 0.008 & 0.0140 & $0.00816 \pm 0.0002$ \\
& 85. & 0.172 & 0.179 & 0.138 & 0.152 & $0.172 \pm 0.008$ \\
& 273. & 1.327 & 1.255 & 1.062 & 1.067 & $1.285 \pm 0.002$ \\
$\mathrm{~N}_{2}$ & 77.7 & 0.0160 & 0.0205 & 0.0128 & 0.0164 & $0.0168 \pm 0.0003$ \\
& 273.2 & 0.181 & 0.183 & 0.145 & 0.146 & $0.185 \pm 0.006$ \\
& 353.2 & 0.287 & 0.286 & 0.230 & 0.228 & $0.287 \pm 0.009$ \\
$\mathrm{CO}_{2}$ & 194.8 & 0.0478 & 0.0557 & 0.0382 & 0.0375 & 0.0516 \\
& 273.2 & 0.0938 & 0.1069 & 0.0750 & 0.0720 & 0.0970 \\
& 312.8 & 0.1218 & 0.1389 & 0.0974 & 0.0935 & 0.1248 \\
& 362.6 & 0.1614 & 0.1847 & 0.129 & 0.1244 & 0.1644 \\
\hline
\end{tabular}

TABLE 2. Diffusion coefficients $\left(\mathrm{cm}^{2} \mathrm{sec}^{-1}\right)$ at 1 atm given by GSS, VSS and experiment.

\begin{tabular}{ccccc}
\hline Gas & $\mathrm{T}\left({ }^{\circ} \mathrm{K}\right)$ & GSS & VSS & Experiment [3] \\
\hline $\mathrm{N}_{2}-\mathrm{H}_{2}$ & 273.2 & 0.696 & 0.712 & 0.674 \\
& 288.2 & 0.764 & 0.783 & 0.743 \\
& 293.2 & 0.787 & 0.808 & 0.76 \\
$\mathrm{~N}_{2}-\mathrm{CO}_{2}$ & 273.2 & 0.134 & 0.120 & 0.144 \\
& 288.2 & 0.148 & 0.131 & 0.158 \\
& 293.2 & 0.153 & 0.135 & 0.16 \\
& 298.2 & 0.157 & 0.139 & 0.165 \\
$\mathrm{H}_{2}-\mathrm{CO}_{2}$ & 273.2 & 0.571 & 0.526 & 0.550 \\
& 288.2 & 0.628 & 0.580 & 0.619 \\
& 293.2 & 0.648 & 0.599 & 0.60 \\
& 298.2 & 0.667 & 0.618 & 0.646 \\
\hline
\end{tabular}


Tables 2 compares the coefficients of diffusion for non-polar molecules given by GSS, VSS and experiment [3], and Table 3 compares those for polar molecule $\mathrm{H}_{2} \mathrm{O}$ and non-polar molecules given by GSS and experiment ${ }^{3}$. The GSS parameters $\varepsilon, \sigma$ and $\delta$ are obtained using the combing laws (25) and (26), respectively. As can be seen, there is an excellent agreement between the GSS and experimental results.

TABLE 3. Coefficient of diffusion $\left(\mathrm{cm}^{2} \mathrm{sec}^{-1}\right)$ for $\mathrm{H}_{2} \mathrm{O}$ and non-polar gases at $1 \mathrm{~atm}$.

\begin{tabular}{cccc}
\hline Gas & $\mathrm{T}\left({ }^{\circ} \mathrm{K}\right)$ & GSS & Experiment [3] \\
\hline $\mathrm{H}_{2} \mathrm{O}-\mathrm{H}_{2}$ & 307.4 & 1.00 & 1.02 \\
& 328.5 & 1.13 & 1.12 \\
& 352.2 & 1.28 & 1.20 \\
$\mathrm{H}_{2} \mathrm{O}-\mathrm{CO}_{2}$ & 307.4 & 0.198 & 0.202 \\
& 328.5 & 0.225 & 0.211 \\
& 352.2 & 0.258 & 0.241 \\
$\mathrm{H} 2 \mathrm{O}-\mathrm{He}$ & 307.4 & 1.00 & 0.90 \\
& 328.5 & 1.12 & 1.01 \\
& 352.2 & 1.26 & 1.12 \\
$\mathrm{H}_{2} \mathrm{O}-\mathrm{N}_{2}$ & 307.4 & 0.272 & 0.256 \\
& 328.5 & 0.309 & 0.303 \\
& 352.2 & 0.352 & 0.359 \\
\hline
\end{tabular}

\section{ACKNOWLEDGEMENT}

This work was supported by Chinese Academy of Sciences. The author would like to acknowledge valuable discussions with Professor Ching Shen.

\section{REFERENCES}

1. Bird, G. A., Molecular Gas Dynamics and the Direct Simulation of Gas Flows, Clarendon, Oxford, 1994.

2. Chapman, S., and Cowling, T. G., The Mathematical Theory of Non-uniform Gases, Cambridge University Press, Cambridge, 1970.

3. Hirschfelder, J. O., Curtiss, C. F., and Bird, R. B., Molecular Theory of Gases and Liquids, Wiley, New York, 1954.

4. Bird, G. A. "Monte Carlo simulation in an engineering context" in Rarefied Gas Dynamics, edited by S. S. Fisher, AIAA, New York, 1981, Part 1, pp.239-255.

5. Bird, G. A. Phys. Fluids 26, 3222-3223 (1983).

6. Koura, K., Matsumoto, H., and Shimada, T., Phys. Fluids A 3, 1835-1837 (1991).

7. Koura, K., and Matsumoto, Phys. Fluids A 3, 2459-2465 (1991).

8. Koura, K., and Matsumoto, H., Phys. Fluids A 4, 1083-1085 (1992).

9. Hassan H. A., and Hash, D. Phys. Fluids A 5, 738-744 (1993).

10. Krieger, F. J., “The viscosity of polar gases,” Project Rand Report RM-646, 1951.

11. Lide, D. R., CRC Handbook of Chemistry and Physics, CRC, New York, 1999-2000. 